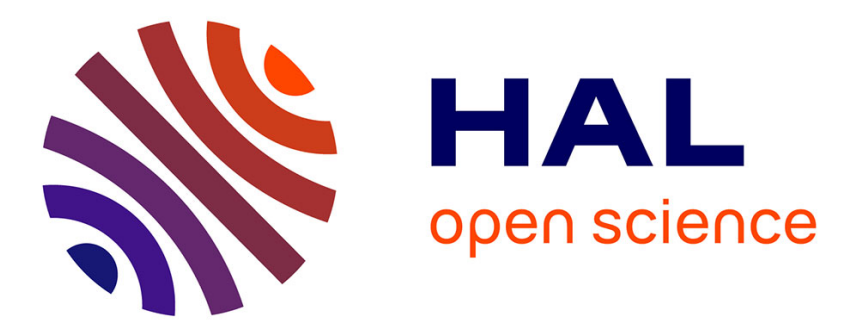

\title{
Propriété intellectuelle et accès public au savoir en ligne
}

Michel Arnaud

\section{To cite this version:}

Michel Arnaud. Propriété intellectuelle et accès public au savoir en ligne. Les Essentiels d'Hermès, 2009, Sociétés de la connaissance. Fractures et évolutions, pp.49-65. halshs-00637074

\section{HAL Id: halshs-00637074 https://shs.hal.science/halshs-00637074}

Submitted on 29 Oct 2011

HAL is a multi-disciplinary open access archive for the deposit and dissemination of scientific research documents, whether they are published or not. The documents may come from teaching and research institutions in France or abroad, or from public or private research centers.
L'archive ouverte pluridisciplinaire HAL, est destinée au dépôt et à la diffusion de documents scientifiques de niveau recherche, publiés ou non, émanant des établissements d'enseignement et de recherche français ou étrangers, des laboratoires publics ou privés. 


\section{PROPRIETE INTELLECTUELLE ET ACCES PUBLIC AU SAVOIR EN LIGNE}

À l'heure de la mondialisation des échanges, un nouveau partage des tâches est organisé entre pays développés et pays en développement, grâce au système des brevets : la conception protégée des produits et services demeure dans les premiers, tandis que la production de masse de type industriel se concentre dans les seconds du fait du différentiel salarial. Avec ce système, les royalties des licences sont versées aux propriétaires des brevets, tandis que les bénéfices de la production industrielle, fondée sur la division du travail et la concurrence, reviennent aux capitaux investis dans les pays émergents. Toutefois, ceux-ci, et l'Inde en particulier, ont appris à leur tour à utiliser le système des brevets à leur profit, créant une pression plus forte chez les industriels de l'immatériel pour prélever leurs revenus dans l'accès contrôlé aux savoirs, grâce au régime de la propriété intellectuelle.

Jusqu'à présent, la fonction des bibliothèques publiques a été d'offrir au grand public le moyen d'accéder aux savoirs à l'aide du support papier, les livres achetés aux éditeurs étant mis à disposition des lecteurs. Avec l'avènement de l'informatique individuelle, ces savoirs deviennent disponibles en ligne, avec la possibilité de les dupliquer sans problème. Cette facilité pourrait jouer un rôle positif dans la diffusion des savoirs et compétences nécessaires à l'amélioration du niveau d'employabilité d'une population confrontée au fonctionnement de la société de l'information, où les employeurs recrutent des salariés capables essentiellement de traiter l'information et de la transformer en savoir.

Mais le régime de la propriété intellectuelle fait que l'accès au savoir en ligne devient payant au nom de la rétribution de l'auteur et des ayants droit que sont le producteur et le diffuseur de contenus. Face à cette offensive tendant à privatiser l'accès à la connaissance, il convient d'analyser les différents niveaux d'offre et de gestion de contenus en fonction du degré de solvabilité des populations concernées. Dans le cas fréquent où celles-ci ne peuvent pas payer les droits de propriété intellectuelle, il y a opposition entre le bien collectif consistant à leur donner les moyens et méthodes pour mieux traiter l'information et les intérêts des entrepreneurs visant à privatiser le plus possible la diffusion des savoirs afin d'en tirer une plus-value. Un arbitrage paraît nécessaire et reste à faire à l'heure actuelle.

Stiglitz propose de faire reconnaître le savoir comme un «bien public international » qui nécessite le soutien des pouvoirs publics. Ce soutien répond aux demandes des populations des pays en développement qui n'ont pas les moyens pour s'équiper à domicile ni encore moins de payer l'accès à des contenus en ligne à des tarifs élaborés par les éditeurs des pays développés. Quelles modalités de mise à disposition de ces contenus seraient à envisager? Quelles mesures prendre pour limiter l'impact négatif des brevets sur l'éducation et la recherche publique ? 


\section{L'accès au savoir en ligne comme bien public international}

Les technologies de l'information et de la communication peuvent permettre à chacun d'entre nous, en tout point du monde, d'accéder quasi instantanément à l'information. Les conditions de mise en place d'une telle facilité impliquent l'utilisation de logiciels libres de moindre coût, le développement des points d'accès public à Internet pour pallier le manque d'équipement informatique à domicile des populations défavorisées mais aussi et surtout la possibilité d'accéder à des contenus libres de droits pouvant répondre à leurs préoccupations.

Si les enjeux concernant les développements informatiques avec les normes ouvertes et les logiciels libres ont été abordés dans les documents de travail du Sommet mondial sur la société de l'information (SMSI), les modalités tendant à la disponibilité gratuite des savoirs restent encore à définir. Elles devraient attirer l'attention des États concernés afin que l'accès au savoir en ligne soit garanti à tous les citoyens, ce qui nécessite un traitement particulier sous formes de subventions spécifiques pour des dispositifs intégrés d'offres en réponse aux besoins exprimés.

\section{Les excès du régime de la propriété intellectuelle}

L'Accord de l'OMC sur les Aspects des droits de propriété intellectuelle qui touchent au commerce (ADPIC), négocié au cours du Cycle de l'Uruguay (Uruguay Round) qui s'est déroulé de 1986 à 1994, a introduit pour la première fois des règles relatives à la propriété intellectuelle dans le système commercial multilatéral. Les droits d'auteur, ainsi que les droits voisins correspondant à ceux de l'éditeur-producteur et du diffuseur, justifient le paiement de redevances lorsqu'un contenu protégé est mis à disposition d'un utilisateur.

On peut noter un glissement au sein du régime de la propriété intellectuelle entre les droits de l'auteur et ceux de l'éditeur-producteur et du diffuseur. Si les droits d'auteur sont la rétribution pour la création d'une œuvre, les droits voisins entrent dans une logique d'appropriation par l'éditeur-producteur et le diffuseur d'une partie du fruit de la vente du bien immatériel, en général supérieure à celle de l'auteur, ceci se justifiant par les coûts de production et de diffusion du bien. Un auteur cède ses droits contre une redevance versée par l'éditeur-producteur et le diffuseur en fonction du chiffre des ventes. Un brevet sous forme de copyright sur une œuvre s'achète et se vend entre éditeurs-producteurs ou diffuseurs en fonction du bénéfice escompté sur les ventes avec le versement des redevances correspondantes. L'auteur s'efface derrière le propriétaire du copyright de son œuvre, dont il touche une petite redevance dans le meilleur des cas.

La finalité des droits d'auteur et du copyright est de garantir à l'auteur un monopole d'exploitation limité dans le temps, en échange de la publication de son œuvre. Mais ce monopole devient de plus en plus long dans un contexte de réattribution de ce droit au propriétaire du brevet. Pour éviter que ne tombent dans le domaine public - c'est-à-dire sans redevance à payer pour leur consultation - des œuvres rendues ainsi accessibles au tout venant, la durée de protection légale du copyright a été allongée aux États-Unis, passant de 14 ans (renouvelable deux fois) en 1790, à 59 ans en 1962, 75 ans en 1976, puis 95 ans en 1998 avec le Mickey Mouse Copyright Act. 


\section{Le verrouillage des outils d'accès aux contenus protégés}

Le traité de l'OMPI (Organisation mondiale de la propriété intellectuelle) de 1996 sur les droits d'auteur (et des ayants droit) donne, dans son article 11, la possibilité à l'auteur de protéger son œuvre par tout dispositif technique approprié et d'en restreindre l'accès et l'usage comme bon lui semble, sans contestation possible.

Aux États-Unis, le traité de l'OMPI de 1996 a été modifié en 1998 dans le cadre du Digital Millennium Copyright Act (DMCA). La doctrine de l'usage privé (fair use), qui garantissait divers droits des utilisateurs (copies privées, citations, etc.), est supprimée. Les ayants droit, c'est-à-dire en pratique les grands éditeurs, décident sans contrainte de la protection des œuvres qu'ils commercialisent. Le texte du DMCA interdit toute publication d'informations pouvant permettre le contournement des mesures techniques de protection.

La Directive européenne sur les droits d'auteur et les droits voisins (European Union Copyright Directive, EUCD), adoptée en 2001, interdit le contournement des mesures techniques de protection et prévoit des amendes et des peines de prison pour les contrevenants. L'acheteur a le droit théorique de faire une copie privée, mais il ne peut pas la réaliser en pratique.

La loi française DADVSI (Droits d'auteurs et droits voisins dans la société de l'information), correspondant à la mise en conformité de la législation française avec la directive européenne EUCD, complétée par la loi Création et Internet (HADOPI) a pour effet principal de restreindre fortement les droits des particuliers achetant des produits culturels. La loi DADVSI s'oppose ainsi directement à la possibilité de réaliser des copies privées en autorisant les producteurs de contenu à mettre en place des mesures techniques les interdisant et surtout en sanctionnant les acquéreurs enclins à contourner ces mesures. Ne pas respecter une mesure de protection, écrire ou diffuser un logiciel permettant de contourner cette protection, ou faire connaître un procédé servant le même but : tous ces actes sont assimilés à des délits de contrefaçon, passibles de prison et d'amende.

Les développements proposés par les entreprises informatiques pour contrôler les ordinateurs des consommateurs afin de vérifier le paiement des droits d'accès aux savoirs sont préoccupants car ils restreignent l'exercice de la liberté d'accès de l'usager. Les principaux producteurs et distributeurs de cinéma et de musique poussent à l'adoption de mesures de contrôle de l'usage et de la copie des œuvres (Digital Rights Management). Elles permettent d'interdire de citer un extrait d'un livre en version électronique reprenant une œuvre pourtant du domaine public. Le nombre de consultations ou d'impressions possibles peut être limité, en bridant techniquement le droit à la copie privée par exemple. Cette approche est mise en œuvre avec l'informatique dite « de confiance » qui consiste à contrôler les usages privés de l'ordinateur par logiciel interposé (Next Generation Computing Base de Microsoft) et par des puces électroniques incluses dans l'équipement informatique (Trusted Computing Group). De cette manière, les producteurs et distributeurs s'assurent que les consommateurs respectent la propriété intellectuelle et paient conformément à leurs exigences. Même lorsqu'un consommateur a acheté une œuvre, ses droits d'accès restent limités à cause des protections imposées par le régime de la propriété intellectuelle. Ces restrictions posent problème quant à la liberté d'usage.

\section{L'accès libre aux contenus comme bien public}

Le rapport au savoir devient plus conflictuel que par le passé car il se trouve au cœur du processus de production des richesses. Dans la société industrielle, les connaissances 
garantissaient la stabilité de l'emploi dans la mesure où celui ou celle qui les possédait se trouvait du côté des cadres et de la maîtrise. Mais, désormais, les savoirs sont fongibles et ne garantissent plus un statut social. Le travailleur intellectuel de la société de l'information doit utiliser son cerveau comme outil de travail afin de transformer les informations en connaissances dans un flux de plus en plus tendu, car la croissance économique est basée sur la production de plus-value immatérielle. La compétition entre nations dans la course aux connaissances nouvelles s'accélère, augmentant la pression sur le travailleur intellectuel.

La consommation payante des savoirs en ligne ne devrait pas être privilégiée au détriment de leur mise à disposition à titre gratuit dans le cadre de l'accès public. Si certains abus de téléchargement abusif donnant lieu à des reventes doivent être réprimés, il n'en reste pas moins que le secteur public devrait jouer un rôle d'arbitre entre, d'une part, l'éditeurproducteur et, d'autre part, le consommateur qui subit un contrôle de plus en plus poussé de ses usages informatiques en vue de possibles prélèvements financiers. Il apparaît nécessaire de revoir la notion sociale de « bien public » comme incluant la mise à disposition de contenus en ligne, libres de droits, au bénéfice de tous. Cela, bien sûr, avec le projet d'une société cohérente qui ne pratiquerait pas de discrimination sur la base du pouvoir d'achat, mais qui au contraire garantirait l'égalité des chances par un accès libre aux savoirs en ligne, permettant d'accroître le niveau général d'employabilité.

Pour contribuer à cet objectif, les logiciels libres et les normes ouvertes sont plus que jamais nécessaires. Ils garantissent la transparence des processus, leur révision étant quasi instantanée en fonction de l'évolution des besoins grâce à la communauté des développeurs, à condition que les normes d'interopérabilité soient respectées. Pour faire face à l'opposition entre les développeurs des logiciels libres et les tenants de l'informatique bridée (qui prétendent répondre aux impératifs de la propriété intellectuelle), il est du ressort des pouvoirs publics de trouver un juste milieu qui permettrait la rétribution des auteurs et éditeurs tout en garantissant une grande marge de manœuvre aux populations défavorisées. En effet, pour que ces dernières restent compétitives, il convient de ne pas les priver de l'accès aux savoirs.

\section{La prise en charge des catalogues de savoirs en ligne par les pouvoirs publics}

Les initiatives telles que l'Open Archive sont intéressantes parce qu'elles concrétisent l'idée de mise à disposition gratuite des savoirs en ligne auprès des utilisateurs. Le «copyleft » doit être aussi encouragé comme résultat de la volonté de l'auteur de dévoiler les contenus qu'il a créés contre une redevance modique. Ce serait le rôle d'organisations telles que l'Unesco d'acheter et de piloter des bases de données correspondant aux besoins des populations défavorisées. Les auteurs et les éditeurs sont dédommagés tandis que leur production est proposée en consultation gratuite au grand public. Le principe de la licence globale est également à soutenir : une redevance mensuelle est prélevée sur les postes d'accès au savoir en ligne et répartie ensuite en fonction des principales sources d'information utilisées.

\section{Le développement des points d'accès public à Internet}

Il ne suffit pas d'offrir des contenus en ligne à des tarifs accessibles ou même de façon gratuite pour garantir l'accès aux savoirs. L'accès public à Internet s'effectue généralement dans les cybercafés dans les pays en développement, dans les télécentres publics dans les pays développés, dans les établissements d'enseignement un peu partout. Or, à partir de cinq à dix 
ans de pratique de ces espaces publics numériques, qui ont contribué à réduire la fracture numérique, il est possible de formuler quelques nouvelles propositions. Ces sites, qu'ils soient des cybercafés, des télécentres ou des lieux d'enseignement, pourraient devenir des foyers de rencontre et de brassage des populations locales, objectif rendu possible par le croisement d'activités de formation et d'information avec des activités ludiques et festives. Dans cette perspective, un accompagnement sous forme d'animation de groupes est à organiser, ce qui implique de former des "infomédiaires ", pédagogues d'un nouveau genre, enseignant les procédures de construction du savoir à partir du traitement de l'information glanée sur les réseaux par les groupes d'apprenants.

\section{Le contrôle des brevets dans les pays en développement}

Les accords bilatéraux ou régionaux sur le commerce et l'investissement conclus entre pays développés et pays en développement comportent souvent des engagements mutuels concernant la mise en œuvre de régimes de propriété intellectuelle (PI) qui vont au-delà des normes minimales prévues dans l'Accord sur les ADPIC. Une pression continue est exercée sur les pays en développement pour qu'ils renforcent la protection de la PI en suivant les normes en vigueur dans les pays développés. Or, la question du mode d'application des brevets aux pays en développement mérite d'être posée. En effet, pour un industriel naviguant dans une économie encore largement informelle, cela n'a pas de sens de déposer un brevet parce que les investissements en recherche et développement sont trop élevés par rapport à son modeste niveau. Par contre, les principaux bénéficiaires des brevets utilisés dans le monde entier sont issus des pays développés et entendent prélever ainsi une dîme sur les économies en développement.

\section{La limitation indispensable du champ d'application des brevets}

Dans le domaine de la santé, le système de la propriété intellectuelle n'a aucun effet incitatif pour la recherche sur des maladies particulièrement répandues dans les pays en développement (paludisme, dengue, etc.), à l'exception des maladies pour lesquelles il existe également un marché important dans le monde développé (le diabète ou les maladies de cœur, par exemple). La mondialisation de la protection de la propriété intellectuelle ne favorise pas la production de médicaments génériques contre le Sida. L'octroi de licences obligatoires est un recours permettant d'obtenir des médicaments brevetés à prix plus bas, selon la déclaration de Doha, mais est-ce une mesure suffisante?

Dans le domaine de la biotechnologie, les paysans des pays en développement n'ont rien à gagner des brevets qui tendent à les spolier de leurs richesses naturelles et de leurs savoirs ancestraux. Ces pays ne devraient pas délivrer de brevets sur les végétaux et les animaux. Ils ne devraient pas approuver le projet de brevetabilité des logiciels : à part l'Inde, aucun de ces pays n'a une masse critique suffisante de produits logiciels exportables. En ce qui concerne les droits d'auteur sur les logiciels, il est clair qu'une application rigoureuse de la loi sur le copyright réduirait le nombre de copies illicites, mais que cela remettrait aussi en question l'accès au savoir des populations défavorisées.

L'OMPI avait accepté d'examiner en octobre 2004 une proposition émanant de l'Argentine et du Brésil, et soutenue par 14 autres pays réunis dans le groupe des «Amis du développement », qui incitait à réexaminer le statut de la propriété intellectuelle afin d'en faire 
un outil de développement et non une fin en soi. Or, les États-Unis et le Japon ont bloqué les négociations. En janvier 2006, le Chili a proposé de protéger davantage l'information dans le domaine public en demandant la reconnaissance de modèles complémentaires de dissémination de la créativité, incluant les logiciels libres. À cette occasion, le Chili a fait remarquer que le régime actuel de la propriété intellectuelle s'opposait aux intérêts nationaux des pays en développement qui paient plus de royalties qu'ils n'en reçoivent. Les États-Unis ont répliqué en proposant d'établir une base de données internationales afin de faciliter l'accès aux brevets. Le Comité du développement et de la propriété intellectuelle (CDIP) créé en 2007 tente depuis l'été 2008 de faire avancer les débats sur la mise en œuvre du Plan d'action de l'OMPI pour le développement.

\section{L'éducation et la recherche publique comme zone franche hors commercialisation des savoirs}

Les institutions d'enseignement et de recherche publique tirent rarement profit d'une politique de dépôt de brevets. Aux États-Unis où ce phénomène est le plus développé, la question du retour sur investissement se pose, selon le rapport de la commission britannique des droits de propriété intellectuelle. Le prix du dépôt des brevets et de leur gestion est à peine inférieur au montant des royalties récoltées. Les équipes d'enseignement et de recherche publique, les apprenants potentiels ont plus à gagner en échangeant librement informations et savoirs (qu'ils se trouvent au Nord ou au Sud) qu'en déposant des brevets. Pour qu'il y ait une éducation et une recherche publique partagées, il faut qu'elles soient hors du champ des brevets. Par exemple, les utilisateurs d'Internet, dans quelque pays que ce soit, devraient pouvoir utiliser de façon raisonnable des copies servant à l'éducation ou à la recherche, et utiliser librement des extraits pour commentaires et critiques. Dans ce cas de figure, toutes les clauses contractuelles ou restrictions techniques imposées par le fournisseur d'informations devraient être nulles et non avenues.

L'accès généralisé au savoir en ligne est une condition indispensable pour un développement humain durable. Aussi, l'argent public doit contribuer à mettre en place, à destination de tous, des bibliothèques et des archives numériques, et des programmes d'enseignement à distance basés sur lnternet. En outre, cela permettra à tous les chercheurs et scientifiques d'accéder en temps réel à des bases de données informatisées comprenant des informations techniques complexes et précises. Néanmoins, pour atteindre un tel but, il faut trouver un compromis avec les producteurs et éditeurs privés, afin que, d'une part, ils ne travaillent pas gratuitement et que, d'autre part, ils ne puissent plus interdire l'accès public au moyen de systèmes de paiement à la séance.

\section{Conclusion}

La brevetabilité de l'immatériel est devenue la base du capitalisme cognitif, principal moteur de croissance dans les pays développés. Elle est critiquée par les chercheurs et les petits industriels de pointe au Nord qui lui reprochent de tuer l'innovation. En effet, toute innovation doit tenir compte de tellement de brevets que cela perturbe sa mise en pratique, à cause de la difficulté à identifier ces brevets et des redevances à payer (qu'il faut ensuite intégrer dans le plan de commercialisation). Les portefeuilles de brevets possédés par les compagnies prennent un caractère spéculatif : ils représentent des options sur l'avenir sous 
forme de licences qui seront accordées ou non aux entreprises. Dans le cas où des licences ne seraient pas accordées, des procès en contrefaçon peuvent être lancés à l'encontre de produits déjà commercialisés ou sur le point de l'être. Pour sa part, le propriétaire du brevet déposé espère que les entreprises vont payer la licence d'exploitation qui leur est proposée, qui représente un coût moindre que d'aller en justice. Cette situation offre, certes, des opportunités aux avocats engagés en vertu d'un contrat à commission finale, aux sociétés concédantes de licences et aux sociétés de conseil qui prétendent aider les entreprises à gérer leurs portefeuilles des brevets ; mais cette manière de procéder tue l'innovation à moyen terme. Lessig, le créateur de Creative Commons, tout comme Stallman, à l'origine de la licence d'exploitation GNU, notent que le partage des savoirs qui a caractérisé l'Internet originel est dénaturé par les tendances actuelles du commerce électronique, de la propriété intellectuelle et de la brevetabilité de l'immatériel.

Les pays en développement n'ont aucune raison d'accepter l'Accord ADPIC dans son état actuel, car ils risquent d'en supporter certaines conséquences assez fâcheuses : paiements aux pays développés de royalties encore plus élevées qu'à présent; entraves dans leurs industries logicielles; soumission des paysans à des brevets qui les ruinent et pillent leurs savoirs ancestraux; interdiction de la fabrication des médicaments génériques alors que les malades en manquent cruellement. Les pays en développement devraient exiger à la fois un contrôle strict du champ d'application des brevets et la mise hors brevet des échanges entre le Nord et le Sud en matière d'éducation et de recherche publique.

L'utilisation de la connaissance comme une denrée qui se vend et s'achète est en contradiction avec la possibilité qu'offrent les nouveaux outils de communication pour accéder à tous les savoirs disponibles sur les réseaux. Il est temps que les États s'engagent dans des négociations internationales pour garantir l'accès au savoir en ligne à tous, en le considérant comme un «bien public international». L'Organisation internationale de la Francophonie pourrait se saisir de ces revendications, qui correspondent non seulement à celles des populations des pays en développement, mais aussi à celles des couches les moins favorisées des pays développés.

\section{RÉFÉRENCES BIBLIOGRAPHIQUES}

COMMISSION BRITANNIQUE DES DROITS DE PROPRIETE INTELLECTUELLE, Intégrer les droits de propriété intellectuelle et la politique de développement, Londres, 2002.

Kaul, I., Grunberg, I., STERn, M., Les Biens publics à l'échelle mondiale : la coopération internationale au XXI siècle, New York et Oxford, Pnud et Oxford University Press, 1999.

LESSIG, L., L'Avenir des idées, Lyon, PUL, 2005.

Perriault, J., L'Accès au savoir en ligne, Paris, Odile Jacob, 2002.

Stiglitz, J., La Grande Désillusion, Paris, Fayard, 2002.

TraOre, A., L'Étau, l'Afrique dans un monde sans frontières, Actes Sud, 1999. 